\title{
Isocitrate Dehydrogenase Inhibitor
}

National Cancer Institute

\section{Source}

National Cancer Institute. Isocitrate Dehydrogenase Inhibitor. NCI Thesaurus. Code C137826.

Any agent that inhibits isocitrate dehydrogenase (IDH). 